\title{
Design and analysis of disc conveyor of cutlery polishing machine
}

\author{
Min $\mathrm{Fu}^{1,2}$, Yuanshuang Chen ${ }^{1,2, *}$, and Loulou Zhang $^{1}$ \\ ${ }^{1}$ College of Mechanical Engineering, Tianjin University of Science \& Technology, Tianjin, 300222, P.R. China \\ 2 Tianjin Key Laboratory of Integrated Design and Online Monitoring for Light Industry \& Food Machinery and Equipment, Tianjin, \\ 300222, P.R. China
}

\begin{abstract}
The paper designs a new disc conveyor of stainless steel cutlery polishing machine. The disc conveyor is mainly composed of transmission mechanism, locating mechanism and clamp mechanism. The paper makes finite element analysis on rotating disc and improves the structure design according to analysis result in order to enhance statics characteristic of rotating disc structure. Through modal analysis, the paper verified the rationality of the design of rotating disc structure. The prototype was put into enterprise and the result showed that this system could reliably operate.
\end{abstract}

\section{Introduction}

The processing of stainless steel cutlery needs to experience more than ten working procedure, of which, polishing is an important link in the production process of stainless steel cutlery. Polishing of stainless steel cutlery can enhance corrosion resistance and smoothness of cutlery, and obtain decorative appearance [1]. The polishing of cutlery is a very complicated technological process and the polishing quality affected the yield of product directly.

Cutlery polishing technology developed earlier in foreign cutlery manufacturers. Most of them adopt fullautomatic polishing machine with high production efficiency and good product quality. Domestic research on cutlery polishing technology starts late. Some small and middle-sized enterprises mainly introduce outdated and obsolete stainless steel cutlery polishing machine from foreign countries, repair and improve it to realize semi-auto production. Large enterprises mainly purchase foreign advanced automatic polisher. However, they fail to master core technology, so the repair and subsequent maintenance cost are high.

With the increasing of labour cost, traditional manual polishing cannot meet the development of enterprise. Domestic cutlery manufacturers increase research spending of automatic polisher. A set of high-automation stainless steel cutlery polishing machine could save labour, reduce the cost and significantly enhance productivity.

According to technological requirement of stainless steel cutlery manufacturers for cutlery polishing, the paper designs a high-automation stainless steel cutlery disc conveyor.
There is a set of five-station cutlery polishing conveyor which is applied to rough grinding of cutlery. Because of fewer configuration and lower production efficiency, which cannot meet the requirement of manufacturers.

According to the disadvantage of polishing conveyor at present, the paper increases number of station according to the program and requirement of manufacturer. We can achieve rough grinding and medium grinding by using one polishing conveyor. The setting of cutlery overturning station and cooling station make the handle and handlebar of cutlery polishing by using a set of conveyor.

\section{The design of cutlery polishing system}

\subsection{General structure layout of cutlery polishing machine}

Cutlery polishing machine is composed of cutlery conveyor that can realize the cutlery intermittent rotational motion, cooling mechanism, cutlery overturning mechanism and layout in the periphery of the eight polishing wax institutions. The transmission mechanism of cutlery polishing machine mainly includes index plate and telescopic cutlery clamping device, Index plate under the drive of the motor to realize the intermittent rotation. It drives extensible clamp mechanism to be transmitted among 12 stations. The function of clamp mechanism is to hold stainless steel cutlery. The polishing and waxing mechanism realizes feeding in transverse and longitudinal direction in the

Corresponding author: 2461216748@qq.com 
polishing process so as to complete cutlery polishing and polishing paste painting in the polishing process. After cutlery polished in four stations, the cooling mechanism cools the cutlery, which avoids the cutlery temperature is exorbitant and appears insufficient clamping force. Cutlery overturning mechanism realizes conversion among cutlery handle and handlebar. The general layout diagram is shown in Fig. 1.

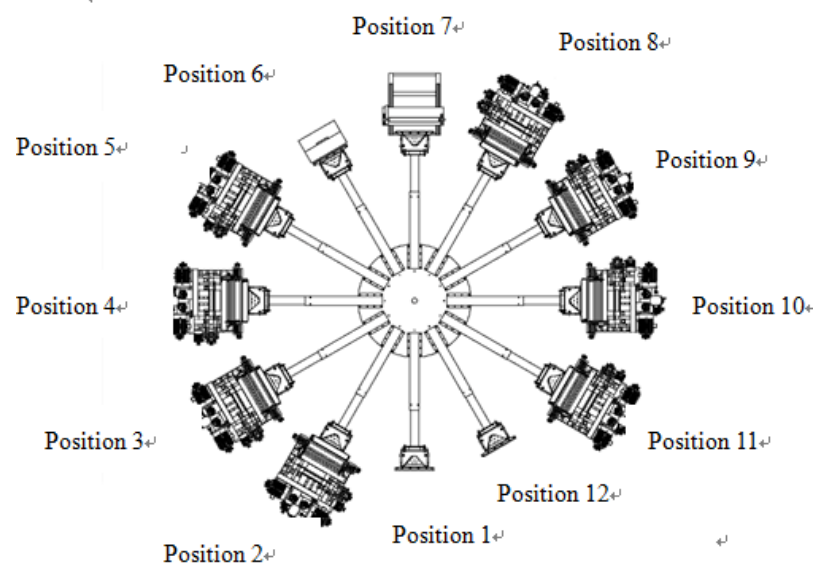

Fig. 1. General structure layout of cutlery polishing machine.

The control action of the whole operation process of cutlery polishing machine is realized by PLC control system. In the actual processing course, the manufacturers can adapt to processing requirement of different kinds of stainless steel cutlery through adjusting transverse and longitudinal feed rate of polishing and waxing mechanism and cutlery polishing wax institutions and the position of the clamping mechanism. The polishing wax agencies in 2 to 9 position of polishing time can be set in advance. The whole set of device has emergency stop control button, to avoid the unexpected incidents in the process of actual production and processing bring unnecessary losses. Only need one worker to preset the parameter and feed the workpiece of cutlery to be processed in the manufacturing process.

\subsection{Structure design of disc conveyor}

Disc conveyor is mainly composed of bottom plate, shore, transmission mechanism, locating mechanism and clamping mechanism and its structure is shown in Fig. 2.

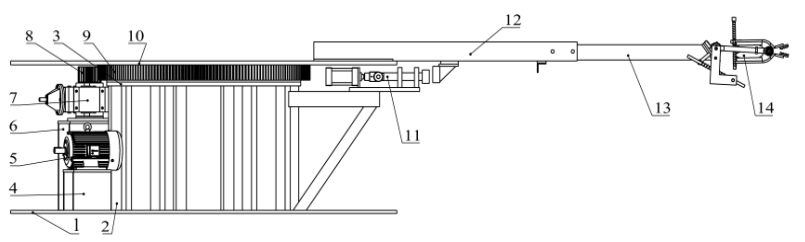

1. Baseplate; 2. Shore; 3. Flange; 4. Motor support; 5 . Motor; 6. Reducer support; 7. Reducer; 8. Small gear; 9. Slewing bearing; 10. Rotary disc; 11. Locating mechanism; 12. Big rectangular steel tube; 13. Small rectangular steel tube; 14. Clamping mechanism

Fig. 2. General structure layout of cutlery polishing machine.
Bottom plate is fixed on the ground using the anchor bolts. The one end of shore on the bottom plate is welded to bottom plate, and the other end is connected to flange. A driving device is fixed on the flange, and the rotating disk is connected with the driving device, and twelve extensible cutlery clamping devices are evenly distributed on the rotating disk, and the fixing frame of the positioning device is connected with the upright post.

\subsubsection{Intermittent motion mechanism}

The core component of the cutlery transmission mechanism is intermittent movement mechanism, and the quality of the intermittent movement mechanism influences the advantages and disadvantages of the cutlery transmission mechanism directly. Many mechanisms can achieve intermittent movement, such as indexing cam mechanism, Maltese mechanism, ratchet mechanism, incomplete gear mechanism, pin wheel mechanism, gear rod compound mechanism, etc. [2]. Through combining technological requirements of actual production, the main transmission system of polisher adopts YVP variable-frequency and variable-speed motor, drives helical gear-spiral bevel gear reducer by belt drive, and passes through small gear of reducer so as to drive rotation of slewing bearing and the rotation of index plate. According to the bearing $(1,000 \mathrm{Kg})$ and maximum rotating speed $(2 \mathrm{r} / \mathrm{min})$ of rotary disc and combining with the design experience, the motor power is $2.2 \mathrm{KW}$ and rated speed is $1440 \mathrm{r} / \mathrm{min}$.

\subsubsection{Pedestal module}

The pedestal of cutlery polishing machine conveyor, on one hand, supports the rotary disc, on the other hand, it is connected to transmission parts and provides power for the rotary disc. The lowest part of the pedestal is baseplate with the diameter of $2,200 \mathrm{~mm}$. There are eight rectangular hollow steel tubes on the baseplate for supporting. The upper part of the steel tube is flange with the thickness of $20 \mathrm{~mm}$ and on the flange is a slewing support. Index plate is connected to rotation support through bolt. Motor drives the rotation of reducer through belt drive. Reducer is connected to small gear, and small gear and rotation bearing realize mesh transmission so as to drives the rotation of rotary disc. Fixed plate of transmission system and fixed plate of locating mechanism are mounted on the pedestal. The structure is shown in Fig. 3.

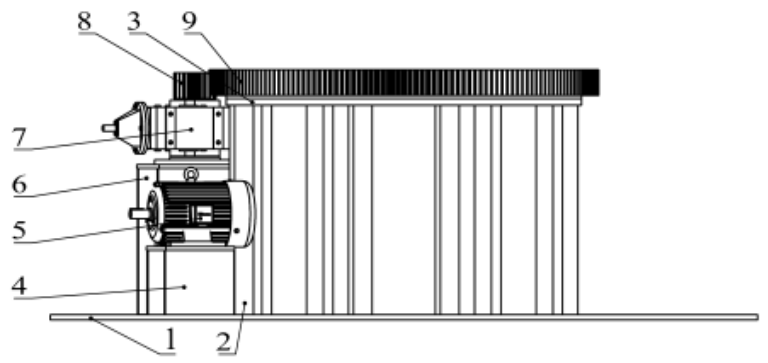

1. Baseplate; 2. Shore; 3. Flange; 4. Motor support; 5 . Motor; 6. reducer support; 7. Reducer; 8. Small gear; 9. Slewing bearing. 
Fig. 3. Pedestal components.

\subsubsection{Locating mechanism}

When the big disc is stopped, the location may be inaccurate owing to inertia, there is a certain deviation. And the polishing machine will have a certain force on the meal in the grinding process of cutlery, the force of the telescopic clamping mechanism by conduction to the chassis causing the chassis vibrating in the grinding station, thereby affecting the polishing quality of cutlery. This paper has designed a set of locating mechanism composed of tip and dead head. On one hand, the mechanism can provide combined force of tip and dead head in horizontal direction; on the other hand, it can ensure tip to keep good guiding orientation in the working process, and its structure is shown in Fig. 4.

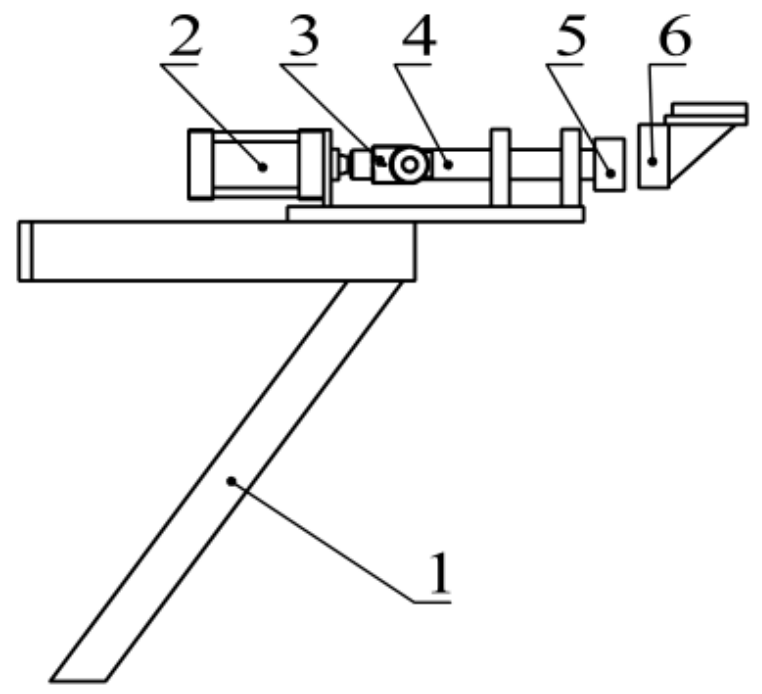

1. support; 2. Air cylinder; 3. Air cylinder contact; 4. Guide shaft; 5. Tip; 6. Dead head.

Fig. 4. Locating mechanism.

\subsubsection{Extensible clamp mechanism}

The cutlery clamp mechanism is an important component of cutlery disc conveyor. When polishing the cutlery, the cutlery often flies off and causes personnel injury because of the high revolving speed of polishing and the low frictional force. The clamp force of pneumatic clamping at the end of such clamp mechanism is provided by rubber air spring. After test analysis, the clamp mechanism can provide enough clamp force and avoid flying-off of cutlery when polishing. Clamp fixture is connected to rotary disc through dead plate, the big rectangular steel tube is welded on the fixed plate, the small rectangular steel tube is installed on the big rectangular steel tube and the small rectangular steel tube is flexible and regulated relative to big rectangular steel tube, the end of small rectangular steel tube is connected to the pneumatic clamp. The structure is shown in Fig. 5.

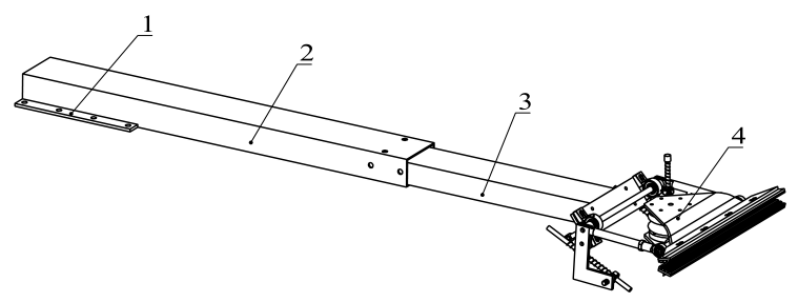

1. Dead plate; 2. Big rectangular steel tube; 3. Small rectangular steel tube; 4 . Pneumatic clamping

Fig. 5. Extensible clamp mechanism.

\section{Finite element analysis and structure improvement of rotating disc}

\subsection{Finite element analysis}

The support stiffness and operational stability of rotary disc are directly related to the polishing quality of cutlery. Therefore, the finite element analysis of rotating disc structure is needed to determine the deformation and stress of the rotating disc under the condition of force.

\subsubsection{Model pretreatment}

The three-dimensional model is built by using 3D software Solidworks, and the model established by Solidworks is saved as $\mathrm{x} \_t$ format file, and then the file is imported into ANSYS Workbench to analyze the linear statics structure. The material is Q235 structural steel, whose modulus of elasticity is $2.0 \times 1011 \mathrm{~Pa}$, poisson's ratio is 0.3 . The model is divided into 81534 nodes and 18520 units by using the method of automatic meshing and the default cell size. Finite element model is shown in Fig. 6.

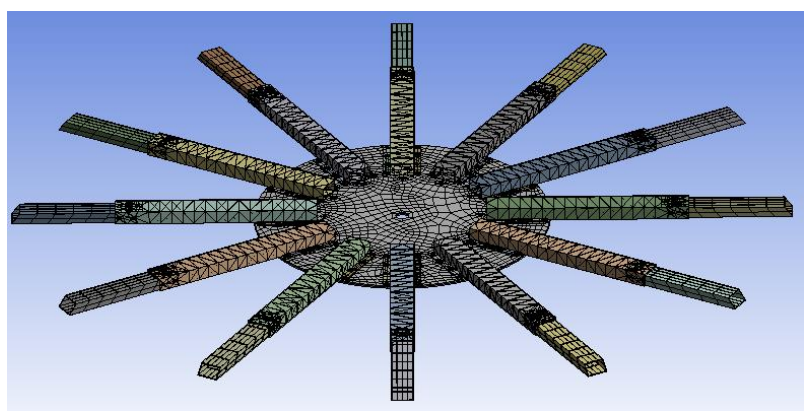

Fig. 6. Finite element model of rotary disc structure.

\subsubsection{Apply load and boundary condition}

Rotary disc and rotary bearing are connected through bolts, so a constraint condition is applied to the 12 bolt holes on the rotating disk, so that the freedom degree is completely restrained. The load applied to the rotary disc mainly originates from the weight of pneumatic clamping and its own weight. After measurement, the weight of each set of pneumatic weight is determined to be about $30 \mathrm{Kg}$; self-weight load mainly includes rotary 
disc, large rectangular steel tube, and small rectangular steel tube. When making finite element analysis, the Standard Earth Gravity is selected, and the uniformly distributed load is applied on each unit at the gravity acceleration of $9.8066 \mathrm{~m} / \mathrm{s} 2$ in ANSYS Workbench [3]. After applying load, the model is shown in Fig. 7.

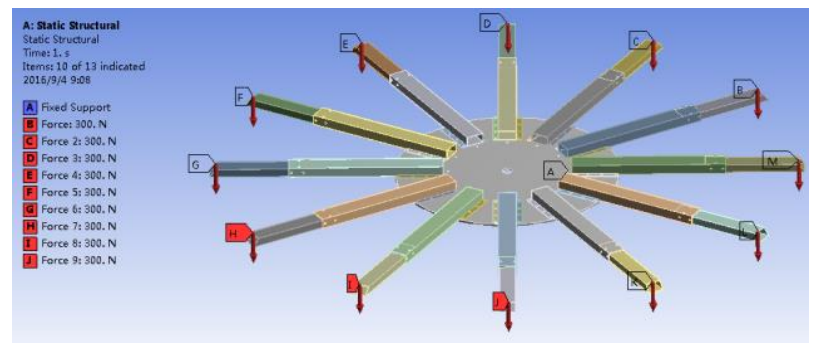

Fig. 7. Schematic diagram of impose restriction and load.

\subsubsection{Result analysis}

The overall deformation diagram and stress nephogram of the rotating disk structure are obtained by solving the finite element model as is shown in Fig. 8.

According to Fig. 8, the deformation between pneumatic clamp and small rectangular steel tube is the largest. The maximum deformation is $7.5032 \mathrm{~mm}$, and the maximum stress is at the connecting bolt hole of rotary disc and rotary bearing. The maximum stress is 288.92Mpa.
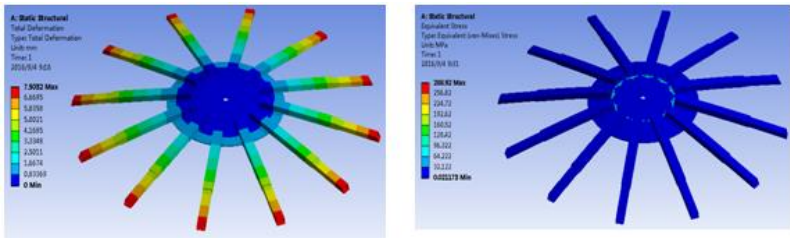

Fig. 8. Overall deformation diagram and stress nephogram of rotary disc structure.

\subsection{The design of structure improvement}

As the basis of conveyor work, the overall deformation of rotary disc structure will affect the polishing quality of cutlery. To reduce overall deformation of rotary disc structure and improve the stress between rotary disc and rotary support connecting bolt hole and enhance the safety factor of equipment, the paper makes structure improvement design of rotary disc structure.

Referring to the design of cable-stayed bridge, the paper determines to set up a cylinder with the diameter of $500 \mathrm{~mm}$, height of $800 \mathrm{~mm}$ and thickness of $10 \mathrm{~mm}$ on a basis of existing structure. The two section $30 \times 30 \times$ $3 \mathrm{~mm}$ square tube is taken as a cable-stayed component. One end of the inclined member is connected with the cylinder, and the other end is connected with the end of the large rectangular steel tube. Two sections of cablestrayed members are connected using regulating threaded rod, and the rotation direction of thread one both ends of threaded rod is opposite so as to ensure movement together of these two sections of cable- strayed members. The modified structure is shown in Fig. 9.

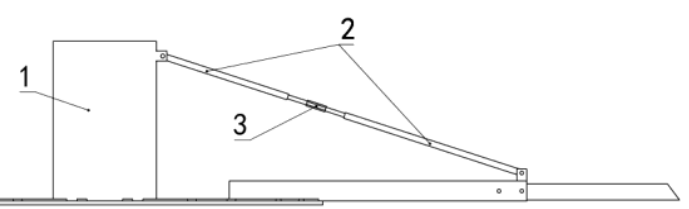

Fig. 9. Schematic diagram of cable-stayed structure.

Static analysis of the improved rotating disk structure model is carried out by using ANSYS Workbench, and the overall deformation and stress nephogram are obtained, as is shown in Fig. 10.
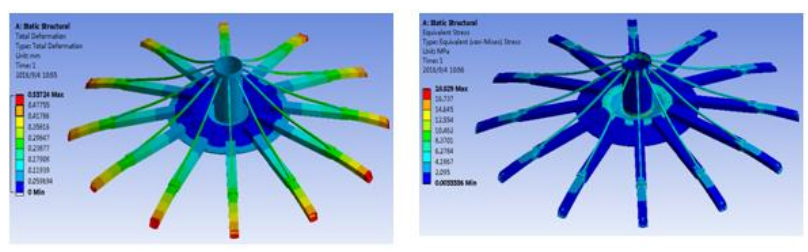

Fig. 10. Overall deformation diagram and stress nephogram after modificaiton.

According to analysis result, it is known the maximum deformation of the modified rotary disc structure is $0.53724 \mathrm{~mm}$, the maximum stress is $18.829 \mathrm{Mpa}$ and the safety factor is 13.728 ; the maximum deformation and maximum stress decline obviously, safety factor is enhanced greatly, stress concentration is improved and stress of rotary disc is distributed around the cylinder evenly. Therefore, the static characteristics of the rotating disc are greatly improved by the improved structure design.

In the process of cutlery polishing, rotary disc structure will produce a certain vibration. During modal analysis, for the one with prestressing force, the inherent frequency is greater than that without prestressing force, and it is more realistic than the actual situation. Thus, the modal analysis of the rotating disc structure is carried out to determine the inherent frequency and vibration mode, so that the external excitation to avoid its natural frequency, to avoid the resonance phenomenon, to ensure stable operation of equipment [4].
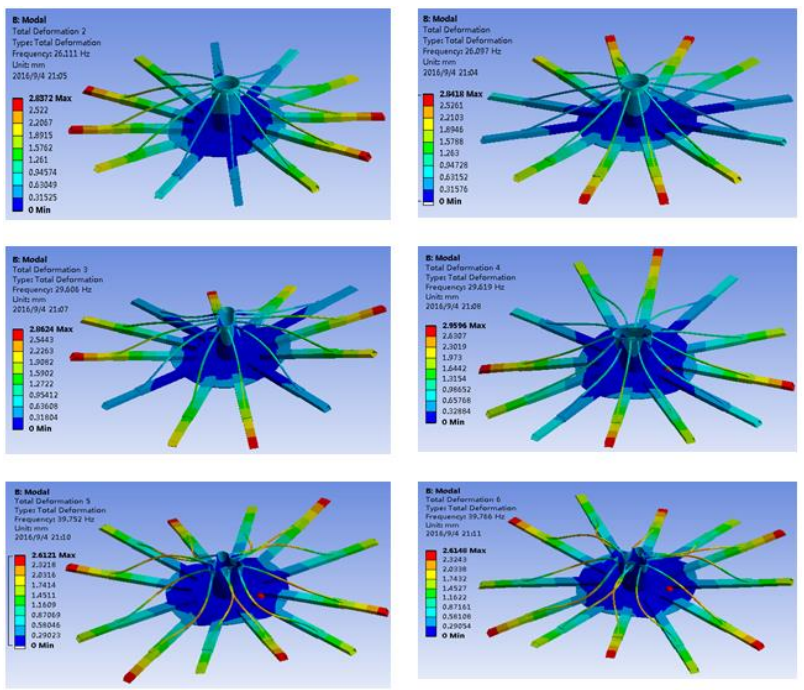
Fig. 11. Mode of vibration and frequency of the first six orders.

On the general conditions, the high-order mode has little effect on the vibration of the structure, usually only consider the low-order mode $[5,6]$. Here, ANSYS Workbench is used to analyze the inherent frequency and mode of vibration of the six orders of rotary disc structure. As shown in Fig. 11.

The modal frequency value and mode of vibration of rotary disc structure at each order as are shown in Table 1.

Table 1. Modal frequency and mode of vibration of the first six orders of rotary disc structure.

\begin{tabular}{|c|c|c|c|c|c|c|}
\hline Order & 1 & 2 & 3 & 4 & 5 & 6 \\
\hline $\begin{array}{c}\text { Inherent } \\
\text { frequency } \\
(\mathrm{Hz})\end{array}$ & 26.097 & 26.111 & 29.606 & 29.619 & 39.752 & 39.766 \\
\hline $\begin{array}{c}\text { Mode } \\
\text { of } \\
\text { vibration }\end{array}$ & $\begin{array}{c}\text { Swing } \\
\text { around } \\
\mathrm{X} \text { axis }\end{array}$ & $\begin{array}{c}\text { Swing } \\
\text { around } \\
\mathrm{Y} \text { axis }\end{array}$ & $\begin{array}{c}\text { Rotate } \\
\text { around } \\
\text { Y axis }\end{array}$ & $\begin{array}{c}\text { Rotate } \\
\text { around } \\
\mathrm{X} \text { axis }\end{array}$ & $\begin{array}{c}\text { Rotate } \\
\text { around } \\
\mathrm{Z} \text { axis }\end{array}$ & $\begin{array}{c}\text { Rotate } \\
\text { around } \\
\mathrm{Z} \text { axis }\end{array}$ \\
\hline
\end{tabular}

According to modal analysis result, the first six order frequency of rotary disc approximates, showing the symmetry of the rotating disk structure. The mode of first six order vibration involves in the swinging and torsion of the overall structure. The inherent frequency range is $26.097 \sim 39.766 \mathrm{~Hz}, 5 \mathrm{~Hz}$ higher than the drive frequency of motor and much lower than the polished roller grinding vibration frequency of $180 \mathrm{~Hz}$. Thus, in the process of cutlery transmission and polishing, the equipment will works safely and reliable which without resonance phenomenon.

\section{Conclusions}

The paper designs a new stainless steel cutlery polishing machine disc conveyor, introduces the mechanical structure composition and work process. The new design has been put into production and used by enterprise. It can polish the predetermined daily 20,000 pieces of the task. The system operates stably and good in polishing effect. It can be popularized and applied to stainless steel cutlery polishing manufacturing industry.

Through modified design of rotary disc structure, the statistical analysis of modified structure by ANSYS Workbench software shows that the rotary disc structure are enhanced greatly in rigidity and strength, also increased the stability of cutlery transmission device which improved the cutlery polishing quality. Moreover, through modal analysis, which verify the rationality of rotary disc structure so as to ensure the equipment operates safely and reliable.

\section{References}

1. J.Y. Hu, J.X. Zhang, M. Fu, Journal of Tianjin University of Sciences \& Technology, 30, 6, 61-65 (2015)
2. M. Fu, L.F. Lin, Machinery Design \& Manufacture, 4, 4, 94-97 (2016)

3. B. Li, Z.J. He, X.F. Chen, ANSYS Workbench design, simulation and optimization (Tsinghua University Press, Beijing, 2013)

4. M. Fu, X. Tang, L.Y. Jin, W. Zhang, Machine Design and Research, 3, 6, 58-61 (2015)

5. Y.X. Wu, J. Han, J.J. Liu, Machinery Design \& Manufacture, 4, 4, 130-132 (2016)

6. H.C. Zhang, Theoretical analysis and engineering application (China Machine Press, Beijing, 2013) 